\title{
Complicaciones obstétricas asociadas al control prenatal inadecuado en puérperas atendidas en un centro materno infantil peruano, 2018
}

\author{
Rebeca M. Núñez-Herrera, Victor H. Moquillaza-Alcántara* y Judith D. García-Cajaleón \\ Servicio de Obstetricia, Centro de Salud Materno Infantil Villa María del Triunfo, Ministerio de Salud, Lima, Perú
}

\section{Resumen}

Objetivo: Evaluar las complicaciones obstétricas asociadas al control prenatal inadecuado en las puérperas atendidās en el Centro Materno Infantil Villa María del Triunfo, en Lima (Perú). Material y métodos: Estudio analítico transversal dondè se evaluaron 66 registros de puérperas atendidas en un centro materno infantil peruano. Se registraron las características sociodemográficas, las complicaciones de acuerdo a la historia clínica y el número de controles prenatales (CPN) de acuerrdo al carné perinatal. Se consideró como control inadecuado aquel que presentó menos de seis CPN o que estos no sẻdistribuyeran durante todos los trimestres del embarazo. El análisis se realizó en STATA, donde se mostraron prevalencias de cada complicación. Las asociaciones fueron evaluadas mediante la prueba Chi cuadrado de Pearson y la razón de prèvalencia, con su respectivo intervalo de confianza al 95\%. La diferencia de medias fue evaluada mediante la prueba $\overline{\bar{T}}$ de Student. Resultados: El 39.39\% de las gestantes presentaron un CPN inadecuado. La inadecuada ganancia de pesळ (IC 95\%: 54.99-78.34\%), infecciones del tracto urinario (IC 95\%: 45.55-69.81\%) y la anemia (IC 95\%: 25.86-49.89\%) fuero complicaciones más prevalentes en gestantes del primer nivel de atención sanitaria. El CPN inadecuado resultó sế un factor de riesgo significativo de infecciones del tracto urinario (OR: 1.54; IC 95\%: 1.03-2.3) y anemia (OR: 1.96; IC 95\%: 3.64). El número de complicaciones obstétricas fue mayor en quienes presentaron un CPN inadecuado $(p=0.008)$. Conclusión: La presencia de anemia e infecciones del tracto urinario se asociaron a CPN inadecuados.

Palabras clave: Atención prenatal. Complicaciones del embarazo. Anemia. Complicaciones infecciosas del embarazo.

\section{Obstetric complications associated with inadequate prenatal control in puerperal women attending a peruvian maternal and child center, 2018}

\section{Abstract}

Objective: To evaluate the obstetric complications associated with inadequate prenatal control in the puerperal women treated at the Maternal and Child Center Villa Maria del Triunfo, in Lima, Peru. Material and methods: Cross-sectional analytical study where 66 records of puerperals attended in a Peruvian maternal and child center were evaluated. The sociodemographic characteristics, the complications according to the clinical history and the number of prenatal controls (NPC) according to the perinatal card were registered. It was considered as inadequate control to those who present $<6$ NPC or that these are not distributed during all the trimesters of pregnancy. The analysis was performed in STATA, where prevalence of each

\section{Correspondencia:}

*Victor H. Moquillaza-Alcántara

E-mail: victor.moquillaza@upch.pe

0187-5337/O 2019. Instituto Naciona licencia CC BY-NC-ND (http://creativecommons.org/licenses/by-nc-nd/4.0/).
Fecha de recepción: 22-04-2019

Fecha de aceptación: 07-11-2019

DOI: 10.24875/PER.19000023
Disponible en internet: 16-12-2019 Perinatol Reprod Hum. 2019;33:58-65 www.perinatologia.mx 
complication was shown. The associations were evaluated using Pearson's Chi-square test and the prevalence ratio, with their respective $95 \%$ confidence interval (CI). The difference of means of communication was made by student's i-test. Results: $39.39 \%$ of the pregnant women presented an inadequate prenatal control. Inadequate weight gain (95\% Cl: 54.99 $78.34 \%$ ), urinary tract infections (95\% Cl: $45.55-69.81 \%$ ) and anemia (95\% Cl: $25.86-49.89 \%$ ) were the most prevallent complications in pregnant women at the first level of pregnancy. Health care inadequate prenatal control was a significeant risk factor for urinary tract infections (OR: 1.54; 95\% Cl: 1.03-2.3) and anemia (OR: 1.96; 95\% Cl: 1.05-3.64). The number of obstetric complications was higher in those who presented inadequate prenatal control $(p=0.008)$. Conclusion: Thespresence of anemia and urinary tract infections were associated with inadequate prenatal controls.

Key words: Prenatal care. Pregnancy complications. Anemia. Infectious pregnancy complications.

\section{Introducción}

La morbilidad obstétrica directa es el resultado de una cadena de acontecimientos adversos durante el embarazo, parto y puerperio; los cuales, de no ser resueltos, pueden concluir en una muerte materna ${ }^{1,2}$. Esta problemática ha venido afectando e incrementándose en muchos países ${ }^{3} \mathrm{e}$ insta a los investigadores a generar revisiones respecto a evidencia que pueda aportar en la anticipación de la muerte en la etapa gestacional ${ }^{4}$. A fin de controlar este proceso, se tiene conocimiento que debe garantizarse una adecuada cobertura y calidad del CPN, definido como la vigilancia y evaluación integral de la gestante y el feto con el objetivo de lograr el nacimiento de un recién nacido sano, sin deterioro de la salud de la madre ${ }^{5-7}$. Este constituye un factor estrechamente ligado a la salud materno-infantil y es, por lo tanto, un área que requiere de investigaciones al respecto que permitan guiar la elaboración de políticas que aporten a la culminación satisfactoria del embarazo ${ }^{8,9}$.

Respecto a la atención, control o cuidado prenatal, ha ido modificándose a lo largo del tiempo, buscando modelos que puedan tamizar oportunamente diversas complicaciones, como aquellos que plantean modelos grupales ${ }^{10,11}$ o previos al embarazo ${ }^{12}$, los cuales han evidenciado resultados alentadores ${ }^{13}$. Sin embargo, aún existen determinantes en el acceso a este servicio, como el pertenecer a poblaciones minoritarias ${ }^{12}$, la ubicación geográfica donde se reside, el bajo desarrollo académico ${ }^{14} \mathrm{O}$ el ingreso económico que perciben las personas $^{15}$. Asimismo, considerando la importancia de que las usuarias acudan a sus controles, se han desarrollado estudios que reportan que la satisfacción en estas atenciones está influida por el acceso, tiempos de espera y el tiempo de atención con el profesional ${ }^{16}$.

Publicaciones previas a nivel internacional ya han mostrado interés por la relación entre el control inadecuado con diversos resultados perinatales. Entre ellos, hay investigaciones que reportan que la ausencia de controles se asocia con hipertensión arterial diastótica, parto prematuro, parto por cesárea y bajo peso a phna$\operatorname{cer}^{17-19}$. Sin embargo, análisis realizados en Áffrica muestran que el impacto no solo se limita al periodo del embarazo, pues se ha determinado que el cumplir con los CPN puede llegar a reducir la probabilidad de muerte neonatal e infantil en un 42.33 y un $30.86 \%$, respectivamente ${ }^{20}$. Por otro lado, en el Perú se observa que del total de muertes maternas, hay un $72 \%$ que presentó $\mathrm{CPN}^{21}$, aunque no se establecen los periödos en los que estos fueron realizados, indicador que para este estudio hemos tomado en cuenta.

Después de las revisiones realizadas sobre el têma se percibe una tendencia de considerar solo el número de controles para establecer que este sea "adeêuado", mas no se evalúa si se realizaron a lo largo del embarazo o se concentran en un trimestre de la gestación ${ }^{22}$. Considerar la distribución del CPN no solo reporta un indicador, sino que permite evaluar la calidad con que se viene realizando, lo cual hace posible establecer acciones preventivas específicas y terapéuticas oportunas durante este periodo. Es preciso indicar que evaluaciones previas ya han determinado que solo el incremento de la cobertura sin garantizar su calidad no mejora los indicadores maternos neonatales $^{23}$. Debido a ello, se plantea como objetivo evałuar las complicaciones obstétricas asociadas al CPN inadecuado en las puérperas atendidas en un centro materno infantil peruano durante el 2018.

\section{Material y métodos}

Estudio analítico transversal donde se evaluarof̧ registros clínicos de puérperas atendidas en el Centro Materno Infantil Villa María del Triunfo, institución sanitaria del Ministerio de Salud perteneciente al primer nivel de atención y ubicada en la región de Lima (Pêrú), durante los meses de enero a abril de 2018. Se incluyeron puérperas que hayan presentado su partö en la institución sanitaria; así mismo, se excluyeron a 
quienes hayan presentado embarazo múltiple o alguna discapacidad mental y/o física que impida su participación en el estudio. No se consideraron los CPN ni diagnósticos de complicaciones realizados en otro establecimiento de salud.

El cálculo del tamaño de la muestra se realizó mediante el programa OpenEpi (http://www.openepi.com/ Menu/OE_Menu.htm), donde se consideró un nivel de confianza del $95 \%$, potencia del $80 \%$, proporción hipotética de controles expuestos del $21.6 \%$ y casos expuestos del $50 \%$, datos obtenidos de un estudio similar realizado en Lima $^{24}$. Generando, así, un total de 51 registros como mínimo para el estudio. El muestreo empleado fue probabilístico, aleatorio simple, en donde se consideró como marco muestral la relación de historias clínicas que tengan registro de alguna atención prenatal en el periodo establecido, escogiéndose al azar hasta cumplir el mínimo de muestra calculado, considerando en todo momento los criterios de inclusión.

Se aplicó la técnica del registro de datos, los cuales fueron obtenidos de las historias clínicas. La variable independiente fue el CPN, en donde se tomó como guía la normativa sanitaria peruana ${ }^{25}$ para considerar como "CPN adecuado» a la gestante que tenga al menos seis CPN, donde dos de ellos se hayan realizado antes de las 22 semanas, el tercero entre la semana 22 y la 24 , el cuarto entre la semana 27 y la 29 , el quinto entre la semana 33 y la 35 y el sexto de la semana 37 hacia adelante. La gestante que no cumplió estas características fue considerada como «CPN inadecuado». Estas consideraciones fueron tomadas de la normativa del Ministerio de Salud del Perú ${ }^{25}$.

Por otro lado, las variables dependientes fueron las complicaciones obstétricas, dimensionadas en complicaciones durante el embarazo (parto pretérmino, infecciones del tracto urinario, vaginosis bacteriana, anemia, oligohidramnios, polihidramnios, desprendimiento prematuro de placenta, preeclampsia, embarazo postérmino, ruptura prematura de membranas, VIH materna, sífilis materna, inadecuada ganancia de peso gestacional, asfixia perinatal, muerte fetal tardía y sufrimiento fetal agudo) y durante el periodo neonatal precoz (macrosomía, bajo peso al nacer, recién nacido postérmino, pequeño para la edad gestacional, grande para la edad gestacional, VIH en recién nacido y sífilis congénita). Las variables mencionadas fueron categorizadas dicotómicamente en: «presenta» y «no presenta». Por otro lado, también se hizo el conteo del número de complicaciones durante el embarazo y durante el periodo neonatal precoz, a fin de generar un subanálisis posterior.
Los registros fueron incluidos en un formato Excel para evaluar la calidad de los datos, luego fueroñexportados hacia el software STATA para su análisis. Las variables descriptivas categóricas se reportaron tmediante frecuencias y porcentajes, mientras que las numéricas, mediante media o mediana (dependieñndo de la normalidad de los valores) y sus medidas de dispersión (desviación estándar o rango intercuartílico). La normalidad fue evaluada mediante la pruebà de Shapiro-Wilk, considerando como distribución normal si el valor $p$ fue mayor a 0.05 . La asociación entrêsariables fue estudiada mediante la prueba Chi cuadrado de Pearson, considerando un nivel de confianza del $95 \%$ y una significancia de 0.05 . Así mismo, se asưmió como asociación significativa cuando se halló un vălor p menor a 0.05 . El riesgo fue evaluado mediante la razón de prevalencia (RP), junto a su intervalo de confianza al 95\%. Finalmente, la diferencia entre $=$ las medias del número de complicaciones entre ambos grupos de interés (CPN adecuado e inadecuado) fue evaluada mediante la prueba $T$ de Student para vafienzas iguales, considerando también un nivel de confilanza del $95 \%$.

El estudio, al no requerir la participación directa de seres humanos, no necesitó la aplicación de un çonsentimiento informado; asimismo, no se hallaron dăños potenciales durante el estudio. No se estipuló ningüún pago a las personas que participaron o cooperaroncon la investigación, ni siquiera los autores. Toda la información obtenida fue codificada y no se registró ningún dato que permita la identificación de las participantes, y se mantuvo toda la información en la computadora personal de la investigadora principal. El estudio fue revisado y aprobado inicialmente por el Comité dē investigación de la Escuela de Obstetricia de la Universidad Nacional Mayor de San Marcos y próximamênte por la dirección del Centro Materno Infantil Villa Maráía del Triunfo.

\section{Resultados}

El estudio incluyó un total de 66 registros clínicos de gestantes atendidas en el Centro Materno Infantil Zilla María del Triunfo durante 2018. En la tabla 1 se observa que la edad promedio fue de $26.4 \pm 5.6$ años- en donde el $33.33 \%(n=22)$ tuvo entre 26 a 30 añosy el $28.79 \%(n=19)$ entre 21 a 25 años. Respecto al estado civil, el $83.33 \%(n=55)$ reportó ser conviviente $\frac{\pi}{y}$ el $10.61 \%(n=7)$ ser soltera. Finalmente, al consultar sobre el grado de instrucción, se obtuvo que el $60.61 \%$ $(n=40)$ había culminado la secundaria completa y el 
Tabla 1. Características sociodemográficas de gestantes que realizan sus controles prenatales en un centro materno infantil peruano, 2018

\begin{tabular}{|l|c|c|}
\hline & n & $\%$ \\
\hline Edad (años) & & \\
15 -20 años & 13 & 19.70 \\
$21-25$ años & 19 & 28.79 \\
$26-30$ años & 22 & 33.33 \\
$31-35$ años & 7 & 10.61 \\
$36-40$ años & 4 & 6.06 \\
$41-45$ años & 1 & 1.52 \\
(Media; DS) & $(26.41 ; 5.63)$ & \\
Distrito de procedencia & & \\
Villa María del Triunfo & 66 & 100.00 \\
\hline Estado civil & & \\
Casada & 4 & 6.06 \\
Conviviente & 55 & 83.33 \\
Soltera & 7 & 10.61 \\
Grado de instrucción & & \\
Ninguno & & \\
Primaria incompleta & 3 & 4.55 \\
Primaria completa & 2 & 3.03 \\
Secundaria incompleta & 3 & 4.55 \\
Secundaria completa & 13 & 19.70 \\
Superior técnico incompleto & 40 & 60.61 \\
Superior técnico completo & 1 & 1.52 \\
Superior universitario incompleto & 2 & 3.03 \\
Superior universitario completo & 1 & 1.52 \\
Total & 66 & 1.52 \\
\hline
\end{tabular}

DS: desviación estándar.

4.55\% ( $\mathrm{n}=3$ ) no presentaba ningún tipo de estudio. La muestra evaluada residía en el mismo distrito (Villa María del Triunfo), el cual se encuentra al sur de Lima.

Al evaluar los registros sobre la atención durante el embarazo se halló que el 39.39\% $(n=26)$ de las gestantes presentaron un CPN inadecuado. La tabla 2 muestra la prevalencia de complicaciones durante el embarazo y el periodo neonatal precoz. Entre las más frecuentes durante el embarazo se halló que el $66.67 \%$ (IC 95\%: 54.99-78.34\%) presentó una ganancia inadecuada de peso gestacional, el 57.58\% (IC 95\%: 45.55$69.81 \%)$ mostró infecciones del tracto urinario y el 37.88\% (IC 95\%: 25.86-49.89\%) presentó anemia. Asimismo, en el periodo neonatal precoz, el $6.06 \%$ (IC 95\%: $0.14-11.97 \%$ ) de los neonatos fueron pequeños para la edad gestacional y el $4.55 \%$ (IC 95\%: 0-9.71\%) presentaron macrosomía y fueron grandes para la edad gestacional.

En la tabla 3 se observan las complicaciones registradas durante el embarazo de acuerdo al CPN adecuado e inadecuado. Los resultados mostraron que quienes tuvieron un CPN inadecuado presentaron una mayor prevalencia de parto prematuro $(7.69 \% ; p=0.352)$, infecciones del tracto urinario $(73.08 \% ; p=0.037)$, anemia $(53.85 \% ; p=0.034)$, ruptura prematura $d e$ membranas (3.85\%; $p=0.211)$ e inadecuada ganancia de peso gestacional (69.23\%; $p=0.729)$. Asimismo, el CPN inadecuado se mostró como factor de riesgo àsociado $(\mathrm{p}<0.05)$ a la infección del tracto urinario \&RP: 1.54; IC 95\%: 1.03-2.30) y anemia (RP: 1.96; IO 95\%: 1.05-3.64).

En la tabla 4 se observan las complicaciones registradas en el periodo neonatal precoz de acuerdo al CPN adecuado e inadecuado. Dentro del grupo de gestantes con CPN inadecuado se hallaron casos de macrosomía (7.69\%; $p=0.352)$, pequeños para la edad gestacional $(3.85 \% ; p=0.556)$ y grandes para la edad gestacional $(7.69 \% ; p=0.352)$, aunque sin una diferencia estadísticamente significativa en comparación el grupo control.

En la tabla 5 se evaluó el número de complicaciones de acuerdo al CPN adecuado o inadecuado. Se enčntró que la cantidad de complicaciones durante losఫtres periodos, denominado como "general», fue significeativamente mayor $(p<0.05)$ cuando la gestante presenta $\mathrm{CPN}$ inadecuados. Asimismo, el número de complicaciones durante el embarazo es significativamènte mayor $(p<0.05)$ cuando la gestante presenta $\overline{G P N}$ inadecuados. Con respecto al número de complicáciones durante el periodo neonatal, este fue mayor cuando la gestante presenta CPN inadecuados, aunque no llega a ser significativamente diferente con respecto a quienes sí presentaron un CPN adecuado.

\section{Discusión}

La importancia de llevar CPN adecuados generaque el profesional de la salud pueda promover actividades preventivas y de promoción en la gestante. La última Encuesta Demográfica y de Salud Familiar (ENDES) en el Perú ${ }^{26}$ reporta que aumentaron al $88.9 \%$ los nnacimientos que recibieron seis o más CPN, lo cuâb difiere con lo hallado en la presente investigación, gue encontró que solo el $60.61 \%$ cumplió con estos. Es preciso indicar que para este estudio se conside é el criterio de la Norma Técnica de Salud para la Atençión Integral de Salud Materna del Ministerio de Salud peruano ${ }^{25}$, la cual no solo considera cumplir con los seis controles mínimos, sino que estos se distribuyan en todos los trimestres del embarazo.

La evaluación de las complicaciones fueron clasificadas en dos categorías: embarazo y periodo neônatal. Con respecto a las complicaciones del embarazo, 
Tabla 2. Prevalencia de complicaciones registradas en un centro materno infantil peruano, 2018

Durante el embarazo
Parto pretérmino
Infecciones del tracto urinario
Vaginosis bacteriana
Anemia
Oligohidramnios
Polihidramnios
Desprendimiento prematuro de la placenta
Preeclampsia
Embarazo postérmino
Ruptura prematura de membranas
VIH
Sífilis
Inadecuada ganancia de peso gestacional
Asfixia perinatal
Muerte fetal tardía
Sufrimiento fetal agudo
Durante el periodo neonatal precoz
Bajo peso al nacer
Macrosomía
Recién nacido postérmino
Pequeño para la edad gestacional
Grande para la edad gestacional
VIH en el recién nacido
Sífilis congénita

\section{n}

3

38

0

25

1

0

0

0

1
0

0

44

44

0

0

1
3
0
4
3
0
0

(IC 95\%)

(0-9.71)

(45.55-69.81)

57.58

0

37.88

1.52

0

0

0

0

1.52

0

66.67

0
0

0
0

1.52

4.55

0

6.06

4.55

0
(25.86-49.89)

(0-4.54)

$-$

$-$

-

(0-4.54)

-

(54.99-78.34)

$-$

-

(0-4.54)

(0-9.71)

(0.14-11.97)

(0-9.71)

*Prevalencia: Proporción de gestantes que presentaron la complicación.

Tabla 3. Complicaciones durante el embarazo asociadas al control prenatal inadecuado en un centro materno infantil peruano, 2018

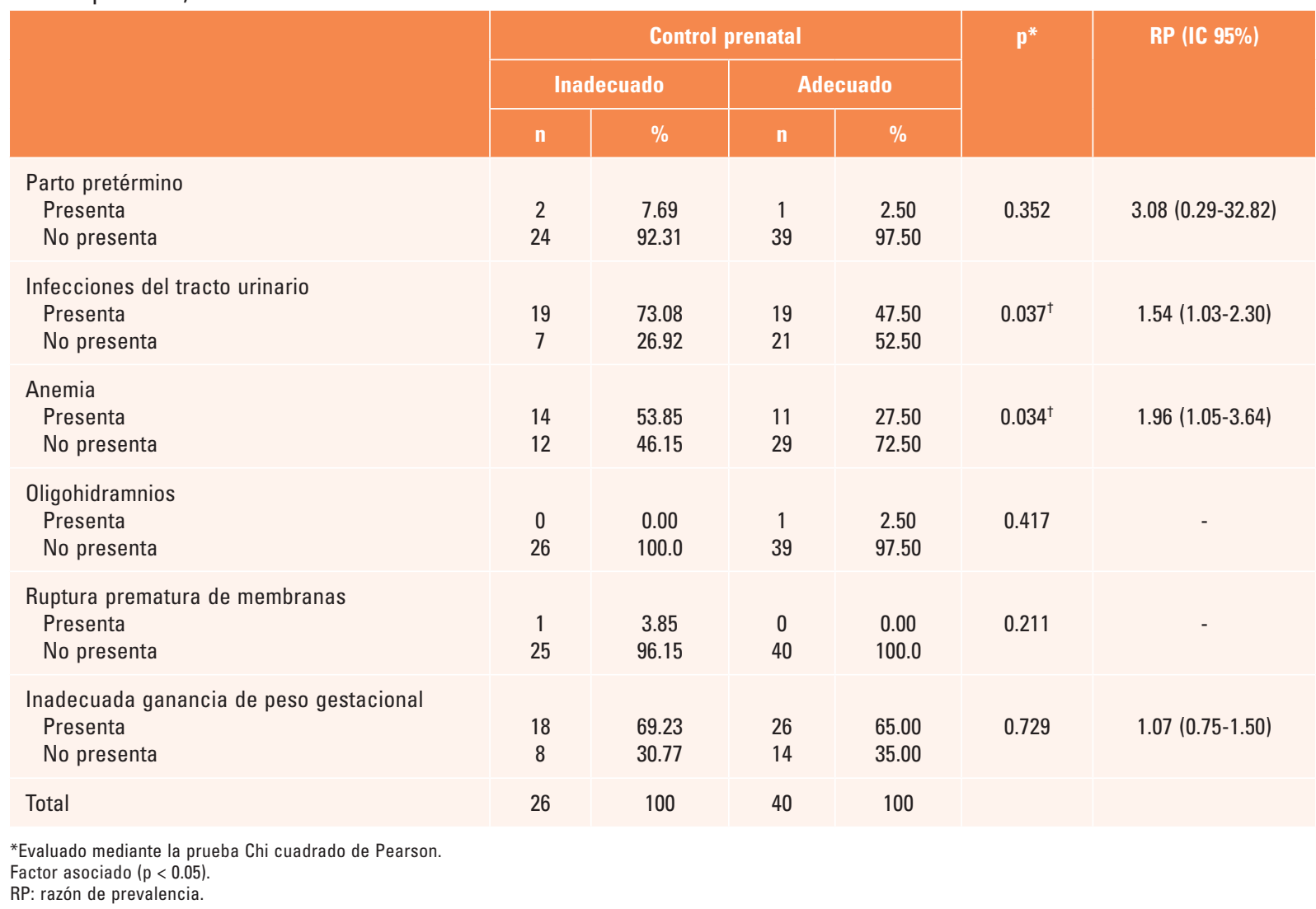


Tabla 4. Complicaciones durante el periodo neonatal precoz asociadas al control prenatal inadecuado en un centro materno infantil peruano, 2018

\begin{tabular}{|c|c|c|c|c|c|c|c|}
\hline & \multicolumn{4}{|c|}{ Control prenatal } & \multirow[t]{3}{*}{$\mathbf{p}^{*}$} & \multirow[t]{3}{*}{ RP (IC 95\%) } & 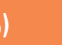 \\
\hline & \multicolumn{2}{|c|}{ Inadecuado } & \multicolumn{2}{|c|}{ Adecuado } & & & \\
\hline & n & $\%$ & $n$ & $\%$ & & & ( \\
\hline $\begin{array}{l}\text { Bajo peso al nacer } \\
\text { Presenta } \\
\text { No presenta }\end{array}$ & $\begin{array}{c}0 \\
26\end{array}$ & $\begin{array}{c}0.00 \\
100.0\end{array}$ & $\begin{array}{c}1 \\
39\end{array}$ & $\begin{array}{c}2.50 \\
97.50\end{array}$ & 0.417 & - & $\begin{array}{l}\overline{0} \\
\dot{c} \\
\dot{0}\end{array}$ \\
\hline $\begin{array}{l}\text { Macrosomía } \\
\text { Presenta } \\
\text { No presenta }\end{array}$ & $\begin{array}{c}2 \\
24\end{array}$ & $\begin{array}{c}7.69 \\
92.31\end{array}$ & $\begin{array}{c}1 \\
39\end{array}$ & $\begin{array}{c}2.50 \\
97.50\end{array}$ & 0.352 & $3.08(0.29-32.8$ & ${ }^{82 \sqrt{\frac{1}{2}}}$ \\
\hline $\begin{array}{l}\text { Pequeño para la edad gestacional } \\
\text { Presenta } \\
\text { No presenta }\end{array}$ & $\begin{array}{c}1 \\
25\end{array}$ & $\begin{array}{c}3.85 \\
96.15\end{array}$ & $\begin{array}{c}3 \\
37\end{array}$ & $\begin{array}{l}7.50 \\
92.50\end{array}$ & 0.556 & $0.51(0.06-4.7$ & $\left.75\right|_{\circlearrowright} ^{\circlearrowleft}$ \\
\hline $\begin{array}{l}\text { Grande para la edad gestacional } \\
\text { Presenta } \\
\text { No presenta }\end{array}$ & $\begin{array}{c}2 \\
24\end{array}$ & $\begin{array}{c}7.69 \\
92.31\end{array}$ & $\begin{array}{c}1 \\
39\end{array}$ & $\begin{array}{c}2.50 \\
97.50\end{array}$ & 0.352 & $3.08(0.29-32.8$ & $\frac{\sqrt{\frac{1}{\sigma}}}{\sqrt{\frac{1}{5}}}$ \\
\hline Total & 26 & 100.0 & 40 & 100.0 & & & 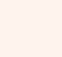 \\
\hline $\begin{array}{l}\text { *Evaluado mediante la prueba Chi cuadrado } \\
\text { Factor asociado }(p<0.05) \text {. } \\
\text { RP: Razón de prevalencia. }\end{array}$ & & ntrc & & do & $\operatorname{trc}$ & ntil & 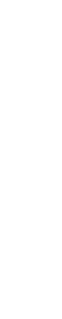 \\
\hline
\end{tabular}

\begin{tabular}{|c|c|c|c|c|c|c|}
\hline & \multicolumn{4}{|c|}{ Control prenatal } & \multirow[t]{3}{*}{$\mathbf{p}^{*}$} & \\
\hline & \multicolumn{2}{|c|}{ Adecuado } & \multicolumn{2}{|c|}{ Inadecuado } & & \\
\hline & Media & DS & Media & DS & & \\
\hline $\begin{array}{l}\text { Complicaciones } \\
\text { General } \\
\text { Durante el embarazo } \\
\text { Durante el periodo neonatal precoz }\end{array}$ & $\begin{array}{c}1.6 \\
1.45 \\
0.15\end{array}$ & $\begin{array}{l}0.14 \\
0.13 \\
0.08\end{array}$ & $\begin{array}{l}2.27 \\
2.07 \\
0.19\end{array}$ & $\begin{array}{l}0.22 \\
0.17 \\
0.11\end{array}$ & $\begin{array}{r}0.008 \\
0.003 \\
0.825\end{array}$ & 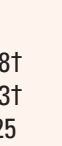 \\
\hline
\end{tabular}

*Evaluado mediante la prueba T de Student para varianzas iguales.

Existe diferencia significativa entre medias $(p<0.05)$.

General: embarazo + periodo neonatal precoz; DS: desviación estándar.

el presente estudio mostró que los CPN inadecuados se asociaron significativamente a las infecciones del tracto urinario y a anemia durante el embarazo. Estos hallazgos coinciden con los resultados de Ruiz, et al. ${ }^{27}$ y López, et al. ${ }^{28}$, realizados en Lima recientemente, donde se reportó que el tener controles de manera inadecuada era un factor de riesgo para presentar anemia materna; así mismo, el primero de ellos ${ }^{27}$ menciona además que la infección del tracto urinario y ruptura prematura de la membrana también están asociados. Es interesante que parte de la evidencia previa se ha realizado en población adolescente ${ }^{27}$, con lo cual se podría estimar que este resultado es independiente de la edad, debido a que se halla la asociación tänto en jóvenes como en adultos.

Por otra parte, estudios en EE.UU. ${ }^{29}$ reportaron que la muerte fetal está asociada al CPN inadecuado; mientras que en estudios latinoamericanos previos ${ }^{30-32}$ se ha evidenciado que un efecto asociado al CPN inădecuado es el parto pretérmino. Ambas complicaciones difieren con lo hallado, por lo que se recomienda generar más estudios a fin de corroborar estos resultados.

Respecto a los resultados del periodo neonataf, el estudio no mostró factores asociados significativamente. Sin embargo, la macrosomía y el ser grande para la edad gestacional fueron condiciones que se reportaron 
mayormente en madres con CPN inadecuado. Ello difiere con estudios previos en donde, por ejemplo, Albuquerque $^{32}$ reporta que el bajo peso al nacer es aquel factor asociado al inadecuado control. Por otro lado, un estudio realizado en Lima $^{24}$ reporta como resultados neonatales del CPN inadecuado a la sepsis neonatal y al Apgar menor a 7. Tal como puede observarse, existe variedad de resultados al respecto de aquellas complicaciones neonatales atribuidas al CPN inadecuado. Sería conveniente que un próximo estudio los aborde, con el fin de generar una conclusión al respecto.

Además, se deseó buscar si la cantidad de complicaciones (evaluada mediante número de patologías registradas en cada periodo) variaba de acuerdo al CPN adecuado o inadecuado. Los resultados mostraron que a nivel general, y durante el periodo del embarazo, existía un mayor número de complicaciones en aquellas gestantes que no cumplían con lo establecido por la Organización Mundial de la Salud y por el Ministerio de Salud del Perú, independientemente de la patología que se evalúe. Esto llega a ser un problema para la salud pública, debido a que es el primer nivel de atención aquel sistema que recibe la información primaria para el manejo y gestión de pacientes, seguimiento asistencial y sistemas de referencia. Esto significa que es aquí donde se deben resolver gran parte de los problemas de salud en las comunidades ${ }^{33}$, lo cual no se llega a cumplir debido a que gran parte de la población no acude al establecimiento y es justamente este grupo quienes finalmente presentan una patología obstétrica, y llegan a ser una carga para el sistema de salud.

Al evaluar las posibles limitaciones encontramos que una de ellas puede ser atribuida al mal registro de ciertas patologías por parte del profesional de la salud, lo cual fue contrarrestado al no solo revisar un documento para fijar una patología, sino corroborarlo con todos los registros clínicos encontrados en la historia clínica. Otro limitante a tener en cuenta es que la investigación fue aplicada en un centro de salud del primer nivel de atención, lo cual implica que las complicaciones halladas en la madre o el feto usualmente son derivadas a instituciones de mayor nivel resolutivo, por lo que existe la probabilidad de que se pierdan registros diagnósticos que aparezcan en el mismo embarazo, parto o puerperio; sin embargo, son situaciones que no pueden modificarse debido a que es el correcto cumplimiento del sistema de salud peruano.

Finalmente, el presente trabajo se distingue de muchos publicados previamente debido a que no solo consideró el criterio de seis controles como mínimo para considerar un CPN adecuado, sino que también abordó en detalle cada semana de gestación; lo cual resulta más preciso debido a que no sirve de mucho tener seis controles si todos fueron realizados duanante los últimos meses. Esperamos que para próximos estudios se aborde este mismo criterio a fin de no coñsiderar a controles inadecuados como adecuados, yque de este modo no se alteren las cifras reportadas. ¿

Con lo cual se concluye que el CPN inadecuado se mostró como factor de riesgo significativo de anemia e infección del tracto urinario durante el embarazo en el Centro Materno Infantil Villa María del Triunfo. Así mismo, las gestantes con CPN inadecuado presentaron mayor frecuencia de neonatos con macrosomita y peso inadecuado para su edad gestacional.

\section{Conflicto de intereses}

Los autores declaran no presentar conflicto de intereses.

\section{Responsabilidades éticas}

Protección de personas y animales. Los autơres declaran que los procedimientos seguidos se conformaron a las normas éticas del comité de experimentación humana responsable y de acuerdo con la Asociación Médica Mundial y la Declaración de Helsinki

Confidencialidad de los datos. Los autores deçaran que han seguido los protocolos de su centrô de trabajo sobre la publicación de datos de pacientes.

Derecho a la privacidad y consentimiento informado. Los autores han obtenido el consentimiento informado de los pacientes y/o sujetos referidos en el artículo. Este documento obra en poder del autor de correspondencia.

\section{Bibliografía}

1. Bendezú G, Bendezú-Quispe G. Caracterización de la morbilidad m̄ăaterna extremadamente grave en un hospital del seguro social del Per氏. Rev Peru Ginecol Obstet. 2014;60(4):291-8.

2. Cook JL, Majd M, Blake J, Barrett JY, Bouvet S, Janssen P, et al Measuring Maternal Mortality and Morbidity in Canada. J Obstet Gynaecol Can JOGC J Obstet Gynecol Can JOGC. 2017;39(11):1028-37.

3. Neggers $\mathrm{YH}$. Trends in maternal mortality in the United States. Reprod Toxicol Elmsford N. 2016;64:72-6.

4. Ozimek JA, Kilpatrick SJ. Maternal Mortality in the Twenty-First Century. Obstet Gynecol Clin North Am. 2018;45(2):175-86.

5. Arispe C, Salgado M, Tang G, González C, Rojas JL. Frecuencia de control prenatal inadecuado y de factores asociados a su ocurrencia. Rev Medica Hered [Internet]. 25 de enero de 2012 [citado 11 de abril de 2019]; 22(4). Disponible en: http://www.upch.edu.pe/vrinve/dugic/revistas/index.php/RMH/article/view/1072.

6. Rico-Venegas RM, Ramos-Frausto VM, Martínez PC. Control prenatal vs resultado obstétrico perinatal. Enferm Glob. 2012;11(27):397-407.

7. Susana-Aguilera P, Peter-Soothill MD. Control Prenatal. Rev Mèdica Clínica Las Condes. 2014;25(6):880-6.

8. Organización Mundial de la Salud. La OMS señala que las embarazadas deben poder tener acceso a una atención adecuada en el momento adecuado [Internet]. [citado 11 de abril de 2019]. Disponible en: bittps:// www.who.int/es/news-room/detail/07-11-2016-pregnant-women-must-beable-to-access-the-right-care-at-the-right-time-says-who. 
9. Organización Mundial de la Salud. Recomendaciones de la OMS sobre la atención prenatal para una experiencia positiva del embarazo [Internet]. [citado 11 de abril de 2019]. Disponible en: https:// apps.who.int/iris/bitstream/handle/10665/250802/WHO-RHR-16.12spa. pdf;jsessionid=2F992F23FC344F6038C433AF0D2D68CE? sequence $=1$

10. Tilden EL, Hersh SR, Emeis CL, Weinstein SR, Caughey AB. Group prenatal care: review of outcomes and recommendations for model implementation. Obstet Gynecol Surv. 2014;69(1):46-55.

11. Picklesimer A, Heberlein E, Covington-Kolb S. Group prenatal care: has its time come? Clin Obstet Gynecol. 2015:58(2):380-91.

12. Gadson A, Akpovi E, Mehta PK. Exploring the social determinants of racial/ethnic disparities in prenatal care utilization and maternal outcome. Semin Perinatol. 2017:41(5):308-17.

13. Gennaro S, Melnyk BM, O\&Connor C, Gibeau AM, Nadel E. Improving Prenatal Care for Minority Women. MCN Am J Matern Child Nurs. 2016;41(3):147-53.

14. Heaman MI, Martens PJ, Brownell MD, Chartier MJ, Thiessen KR, Derksen SA. Inequities in utilization of prenatal care: a population-based study in the Canadian province of Manitoba. BMC Pregnancy Childbirth. 2018;18(1):430.

15. Breathett K, Filley J, Pandey M, Rai N, Peterson PN. Trends in Early Prenatal Care Among Women with Pre-Existing Diabetes: Have Income Disparities Changed? J Womens Health 2002. 2018;27(1):93-8.

16. Adeyinka O, Jukic AM, McGarvey ST, Muasau-Howard BT, Faiai M, Hawley NL. Predictors of prenatal care satisfaction among pregnan women in American Samoa. BMC Pregnancy Childbirth. 2017;1 $7(1): 381$.

17. Liu C-M, Chang S-D, Cheng PJ. Relationship between prenatal care and maternal complications in women with preeclampsia: Implications for continuity and discontinuity of prenatal care. Taiwan J Obstet Gynecol. 2012:51(4):576-82.

18. Huang A, Wu K, Zhao W, Hu H, Yang Q, Chen D. Attendance at prenatal care and adverse birth outcomes in China: A follow-up study based on Maternal and Newborn's Health Monitoring System. Midwifery. 2018; 57:26-31.

19. Nimi T, Fraga S, Costa D, Campos P, Barros H. Prenatal care and pregnancy outcomes: A cross-sectional study in Luanda, Angola. Int J Gynaecol Obstet Off Organ Int Fed Gynaecol Obstet. 2016;135 Suppl 1: S72-8.

20. Makate $\mathrm{M}$, Makate $\mathrm{C}$. The impact of prenatal care quality on neonatal, infant and child mortality in Zimbabwe: evidence from the demographic and health surveys. Health Policy Plan. 2017;32(3):395-404.

21. Centro Nacional de Epidemiología, Prevención y Control de Enfermedades. Vigilancia epidemiológica de la muerte materna en el Perú. [Internet]. [citado 11 de abril de 2019]. Disponible en: http://www.dge.gob.pe/ portal/docs/tools/teleconferencia/SE012017/05materna.pdf.
22. Coley SL, Zapata JY Schwei RJ, Mihalovic GE Matabele MN Jacobs EA More than a "Number": Perspectives of Prenatal Care Quality from Mothers of Color and Providers. Womens Health Issues Off Publ Jacobs Inst Womens Health. 2018;28(2):158-64.

23. Ahmed M, Won Y. Cross-National Systematic Review of Neonatal'Mortality and Postnatal Newborn Care: Special Focus on Pakistan Int J Environ Res Public Health. 2017;14(12). pii: E1442.

24. Alberti I, Lenyi F. Controles Prenatales y Puntaje de Apgar menor de Tecomo Determinantes de Sepsis Neonatal Temprana. Hospital Nacional Dos de Mlayo. 2014. Univ Priv Antenor Orrego - UPAO [Internet]. 2015 [citado 6 de abril de 2019]; Disponible en: http://repositorio.upao.edu.pe/handle/upaorep/1590.

25. Guias nacionales de atención integral de la Salud Sexual y Reproductiva. Ministerio de Salud del Perú [Internet]. [citado 6 de abril de 20191. Disponible en: http://bvs.minsa.gob.pe/local/dgsp/63 guiasnac.pdf

26. INEI - Perú: Encuesta Demográfica y de Salud Familiar 2017 - Nacional y Regional [Internet]. [citado 28 de marzo de 2019]. Disponible en: hittps:// www.inei.gob.pe/media/MenuRecursivo/publicaciones_digitaleS/Est/ Lib1525/index.html.

27. Ruiz M, Ali Y. Principales complicaciones obstétricas del embarazo en gestantes adolescentes del departamento de ginecología y obstetricia del Hospital María Auxiliadora durante el año 2016. Repos Inst - UPSJB [Internet]. 2017 [citado 10 de abril de 2019]; Disponible en: http://repositorio.upsjb.edu.pe/handle/upsjb/841.

28. López R, Steven P. Control prenatal inadecuado como factor de riesgo asociado a anemia materna en el hospital belén de trujillo. Univ Priv Antenor Orrego - UPAO [Internet]. 2018 [citado 10 de abril de 2019]; Disponible en: http://repositorio.upao.edu.pe/handle/upaorep/3945.

29. Monahan R, Caballero A, Reddy U, Stoll B, Silver B, Saade G. 198 : Inadequate prenatal care increases the risk for stillbirth. Am J Obstet Gynecol. 1 de enero de 2018;218(1, Supplement):S131-2. DOI: https:// doi.org/10.1016/j.ajog.2017.10.075.

30. Reyes M, Khiara M. Control prenatal inadecuado como factor asociáa a parto pretermino en pacientes del Hospital Regional Docente de Trujillō. Univ Priv Antenor Orrego [Internet]. 25 de enero de 2017 [citado 10 de abril de 2019]; Disponible en: http://repositorio.upao.edu.pe/handle/upaorep/2249.

31. Ahumada-Barrios ME, Alvarado GF, Ahumada-Barrios ME, Alvarado GF. Risk Factors for premature birth in a hospital. Rev Lat Am Enfermagem [Internet]. 2016 [citado 10 de abril de 2019];24. Disponible en: http:/fwww. scielo.br/scielo.php?script=sci_abstract\&pid=S0104-11692016000-1003 $50 \& \operatorname{lng}=e n \& n r m=i s o \& t \operatorname{lng}=e s$.

32. Gonzaga ICA, Santos SLD, Silva ARV da, Campelo V. Atenção pré-natal e fatores de risco associados à prematuridade e baixo peso ao nascer em capital do nordeste brasileiro. Ciênc Saúde Coletiva. junio de 2016:21 अ96574. DOl: http://dx.doi.org/10.1590/1413-81232015216.06162015.

33. García-Balaguera C, García-Balaguera C. Barreras de acceso y calidad en el control prenatal. Rev Fac Med. junio de 2017; 65(2): 305-10-DOI: https://doi.org/10.15446/revfacmed.v65n2.59704. 